\title{
TIME RESOLVED FLUORESCENCE DIFFUSE OPTICAL TOMOGRAPHY USING MULTI-RESOLUTION EXPONENTIAL B-SPLINES
}

\author{
Nicolas Ducros ${ }^{1,2}$, Anabela Da Silva ${ }^{3,4,5}$, Jean-Marc Dinten ${ }^{1}$, Chandra Sekhar Seelamantula, ${ }^{6}$ \\ Michael Unser ${ }^{6}$, and Françoise Peyrin ${ }^{2}$ \\ ${ }^{1}$ CEA, LETI, MINATEC, 17 rue des Martyrs, F-38054 Grenoble, France. \\ ${ }^{2}$ CREATIS-LRMN, INSERM U 630; CNRS UMR 5220; Université de Lyon; INSA de Lyon, \\ bât. Blaise Pascal, F-69621 Villeurbanne Cedex, France. \\ ${ }^{3}$ Aix-Marseille Université, Institut Fresnel. \\ ${ }^{4}$ CNRS, Institut Fresnel, Campus Saint-Jérôme, F-13013 Marseille. \\ ${ }^{5}$ École Centrale Marseille, Institut Fresnel. \\ ${ }^{6}$ Biomedical Imaging Group, EPFL, CH-1015 Lausanne, Switzerland.
}

\begin{abstract}
This paper deals with the problem of time-resolved fluorescence diffuse optical tomography. We propose a new reconstruction scheme based on a multi-resolution approximation of the time-resolved signals. The underlying basis functions are exponential B-splines that are matched to the decay of fluorescence signals. We illustrate the applicability of the method on phantom data.
\end{abstract}

Index Terms - Fluorescence diffuse optical tomography (FDOT), time-resolved FDOT, exponential B-splines.

\section{INTRODUCTION}

Fluorescence Diffuse Optical Tomography (FDOT) aims at localizing and quantifying fluorescent markers within biological tissue, with light in the near infra-red range. The principle of this method is to use the fluorescence light that exits the medium at different points, and solve an inverse problem to reconstruct the 3-dimensional (3D) marker concentration. This method, which possesses potential application for cancerous tumor detection, is raising increasing interest.

There are three common approaches for performing FDOT acquisitions: continuous wave $(\mathrm{CW})$, frequency domain (FD) and time-resolved (TR). CW FDOT is based on the measurement of the attenuation of a steady state excitation light [1]. FD FDOT is based on the measurement of the phase and amplitude of the modulated excitation light [2]. TR FDOT is based on the temporal measurement of the response to an excitation light pulse $[3,4,5,6,7]$. The $\mathrm{CW}$ and FD approaches are inexpensive and easy to develop, while TR-FDOT is more costly and difficult to implement.

This work has been supported by the Région Rhône-Alpes in the context of project I3M 'Multiscale Medical Imaging and modeling: from the small animal to the human being' of cluster ISLE.
However, the TR approach is potentially able to reconstruct markers embedded as deep as several centimeters within the medium [7]. TR is a practical way of maximizing the amount of information collected from the measurements.

Since the introduction of this modality, researchers have looked for the best way to extract and compress the extra information provided by the TR signals. The focus was initially set on early arriving photons [3]. This approach, however, suffers from low signal-to-noise ratio and provides poor depth resolution. A more recent trend has been to exploit some global features of the time-resolved signals $[4,5,6]$. In particular, researchers have investigated the use of the Laplace transform [4] as well as the moments of the TR fluorescence signals $[5,6]$. However, there is still a crucial need to optimize and compress the extra information provided by the TR signals.

In this paper, we introduce a novel feature extraction process and develop a corresponding reconstruction algorithm. Specifically, the TR signal is described by a sequence of inner products with exponential B-splines [8]. The justification for this type of representation is twofold. First, fluorescence is physically related to exponentially decaying signals that are well represented in terms of exponential splines. Second, it provides a (non-stationary) multiresolution representation where the level of detail is controlled via the knot spacing (or the scale) of the spline [8].

\section{THEORY}

Physically, FDOT is a three-step process. The light is first turned on and it propagates from the source to a given fluorescence marker within the medium. This excites the fluorophore and induces an emission at a higher wavelength (Step 2 ). The emitted fluorescence light then eventually propagates 
from the marker to the detector (Step 3).

\subsection{Time-resolved forward model}

Light propagation within biological tissues is strongly dominated by absorption and scattering of light. Classically, the light propagation in these so-called turbid media is modeled as a diffusion process. This approximation relies on the assumptions that the scattering probability is much larger than the absorption. This assumption, although somewhat restrictive, holds in most practical FDOT scenarios. Moreover, the relative simplicity of the diffusion approximation allows for a significant reduction in the computational costs.

Within the framework of the diffusion approximation, the photon density $\phi(\boldsymbol{r}, t)\left(\mathrm{W} . \mathrm{cm}^{-2}\right)$ at position $\boldsymbol{r}$ and time $t$ can be expressed by means of the following partial differential equation [9]:

$$
-\nabla D(\boldsymbol{r}) \nabla \phi(\boldsymbol{r}, t)+\frac{1}{v} \frac{\partial}{\partial t} \phi(\boldsymbol{r}, t)+\mu_{a}(\boldsymbol{r}) \phi(\boldsymbol{r}, t)=S(\boldsymbol{r}, t),
$$

where $D(\boldsymbol{r})(\mathrm{cm})$ is the diffusion constant defined by $D=$ $1 /\left(3 \mu_{s}^{\prime}\right) ; \mu_{s}^{\prime}(\boldsymbol{r})\left(\mathrm{cm}^{-1}\right)$ is the reduced scattering coefficient, $\mu_{a}(\boldsymbol{r})\left(\mathrm{cm}^{-1}\right)$ is the absorption coefficient, $v\left(\mathrm{~cm} \cdot \mathrm{ns}^{-1}\right)$ is the speed of light in the turbid medium, and $S(\boldsymbol{r}, t)\left(\mathrm{W} . \mathrm{cm}^{-3}\right)$ is the instantaneous power density of the source.

\subsubsection{Fluorescence}

In this work, the pulse response of the fluorescent markers is modeled by a decaying exponential:

$$
f(\boldsymbol{r}, t)=c(\boldsymbol{r}) \eta \exp [-t / \tau(\boldsymbol{r})] / \tau(\boldsymbol{r}),
$$

where $c(\boldsymbol{r})(\mu \mathrm{M})$ is the local marker concentration at position $\boldsymbol{r}, \tau(\boldsymbol{r})$ (ns) is the fluorescence lifetime, and $\eta$ is the quantum yield. In the following, the lifetime $\tau$ is assumed to be a constant. We denote $e(t)=\eta \exp (-t / \tau) / \tau$.

\subsubsection{FDOT signal}

In the following analysis, the spatial dependence of a function is indicated as a subscript. With this convention, the temporal measurement performed at the detection point $d$ resulting from the excitation of the medium at the source point $s$ is denoted by $m_{\boldsymbol{s}, \boldsymbol{d}}(t)$.

In [10], we have shown that the signal measured by an acquisition setup can be modeled - to a good approximation-by the photon density at the detection point. Consequently, a point marker at position $\boldsymbol{r}_{\boldsymbol{n}}$, with local concentration $c_{n}=$ $c\left(\boldsymbol{r}_{n}\right)$, leads to the following measurement [11]:

$$
m_{\boldsymbol{s}, \boldsymbol{d}}(t)=c_{n}\left(\phi_{\boldsymbol{s}, \boldsymbol{r}_{\boldsymbol{n}}} * e * \phi_{\boldsymbol{r}_{\boldsymbol{n}}, \boldsymbol{d}}\right)(t),
$$

where the symbol $*$ refers to the convolution along the time axis, $\phi_{s, r_{n}}$ denotes the Green's function of the light propagation operator $\mathcal{P}=-\nabla D(\boldsymbol{r}) \nabla+\frac{1}{\nu} \frac{\partial}{\partial t}+\mu_{a}(\boldsymbol{r})$ at point $\boldsymbol{r}_{\boldsymbol{n}}$, the source term being $\delta(\boldsymbol{r}-\boldsymbol{s}) \delta(t)$. Similarly, $\phi_{\boldsymbol{r}_{\boldsymbol{n}}, \boldsymbol{d}}$ is the Green's function of $\mathcal{P}$ at point $\boldsymbol{d}$, the source term being $\delta\left(\boldsymbol{r}-\boldsymbol{r}_{\boldsymbol{n}}\right) \delta(t)$.

Equation (3) is the mathematical description of the three-step physical process of FDOT. $\phi_{\boldsymbol{s}, \boldsymbol{r}_{n}}$ is the time course of the amount of light at position $\boldsymbol{r}_{\boldsymbol{n}}$ due to the pulse excitation at point $\boldsymbol{s}$. The convolution $\left(\phi_{\boldsymbol{s}, \boldsymbol{r}_{n}} * e\right)(t)$ represents the time course of the fluorescence light intensity that is re-emitted at position $\boldsymbol{r}_{n}$ due to the presence of the fluorescent marker. The term $\phi_{\boldsymbol{s}, \boldsymbol{r}_{\boldsymbol{n}}} * e * \phi_{\boldsymbol{r}_{\boldsymbol{n}}, \boldsymbol{d}}$ represents the amount of light leaving the medium from the point $\boldsymbol{d}$ after propagation of the fluorescence light from the point $\boldsymbol{r}_{\boldsymbol{n}}$. This quantity is weighted by the local concentration of the marker to give the actual measurement.

Considering a distribution of the point markers at positions $\left\{\boldsymbol{r}_{\boldsymbol{n}} \in \Omega, n=1,2,3, \ldots, N\right\}$, the effective measurement is given by the sum of the individual contributions. Moreover, considering a distribution of source points $s_{\boldsymbol{i}}, i \in[1, I]$ and detector points $\boldsymbol{d}_{\boldsymbol{j}}, j \in[1, J]$, a set of $I \times J$ measurement pairs can be formed and consequently as many equations. This is written into the form of a matrix equation:

$$
\boldsymbol{m}(t)=\boldsymbol{W}(t) \boldsymbol{c},
$$

where the measurement vector $\boldsymbol{m}(t) \in \mathbb{R}^{I \times J}$ is such that $\boldsymbol{m}(t)=\left[m_{1,1}(t) \ldots m_{i, j}(t) \ldots m_{I, J}(t)\right]^{T}$, and the concentration vector $c \in \mathbb{R}^{N}$ such that $c=\left[c_{1}, \ldots c_{n}, \ldots c_{N}\right]^{T}$. The weight matrix $\boldsymbol{W}(t) \in \mathbb{R}^{(I \times J) \times N}$ that maps the concentration vector to the measurement vector comprises the entries: $w_{(i, j), n}(t)=\left[\phi_{\boldsymbol{s}_{i}, \boldsymbol{r}_{n}} * e * \phi_{\boldsymbol{r}_{n}, \boldsymbol{d}_{j}}\right](t)$.

\section{MATERIAL AND METHODS}

\subsection{Transformation of the TR signals}

In this work we consider a MRA of the TR signals, along the time axis. Explicitly, at scale $i$, we consider a transformation that compresses any signal $s(t)$ to the set of coefficients $\hat{m}_{i}[k]$. We define:

$$
\hat{m}_{i}[k]=\int s(t) \phi_{k, i}(t) \mathrm{d} t,
$$

where $\phi_{k, i}(t)=\beta_{i}\left(t-k 2^{i} T_{s}\right), \beta_{i}$ being an exponential Bspline of order 0 [8]. To tune the basis functions with the physics, we consider an exponential B-spline with an exponential decay corresponding to the fluorescence. Thus, we define $\beta_{i}(t)=\exp (-t / \tau)\left[u(t)-u\left(t-2^{i} T_{s}\right)\right]$, where $u$ is the unit step function, and $\tau$ the fluorescence lifetime. The time step $T_{s}$ is set to $500 \mathrm{ps}$ and the integration is performed over the range $[1,5] \mathrm{ns}$. As a result, card $\left(\hat{s}_{i}[k]\right)=2^{3-i}$.

Applying this transformation component-wise on both sides of (4) leads to the following MRA system:

$$
\hat{\boldsymbol{m}}_{i}=\hat{\boldsymbol{W}}_{i} \boldsymbol{c},
$$

where $\hat{\boldsymbol{m}}_{i}$ and $\hat{\boldsymbol{W}}_{i}$ are the concatenation of the $\hat{\boldsymbol{m}}_{i}[k]$ 's and the $\hat{\boldsymbol{W}}_{i}[k]$ 's, respectively. 


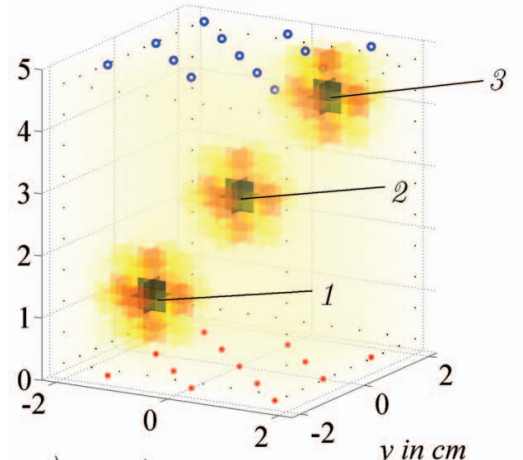

a) $\quad x$ in $\mathrm{cm}$
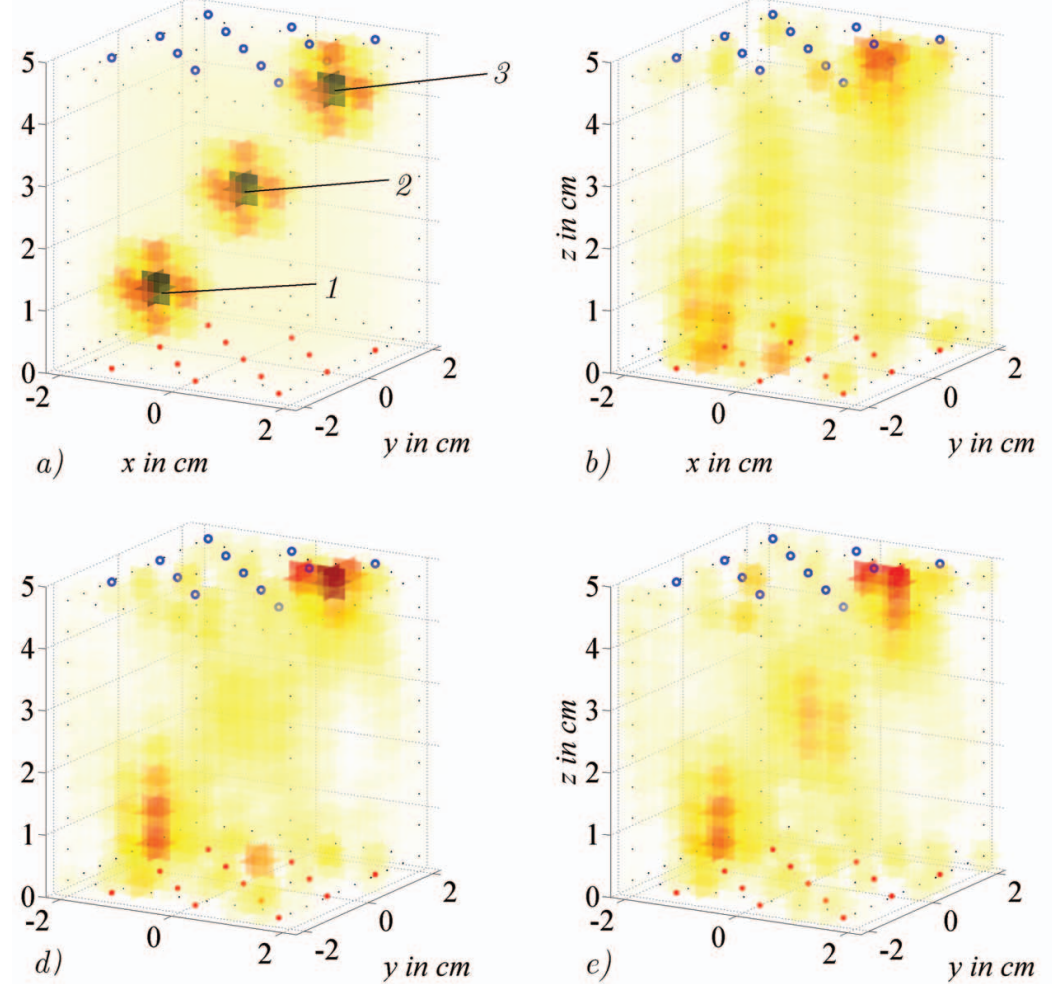

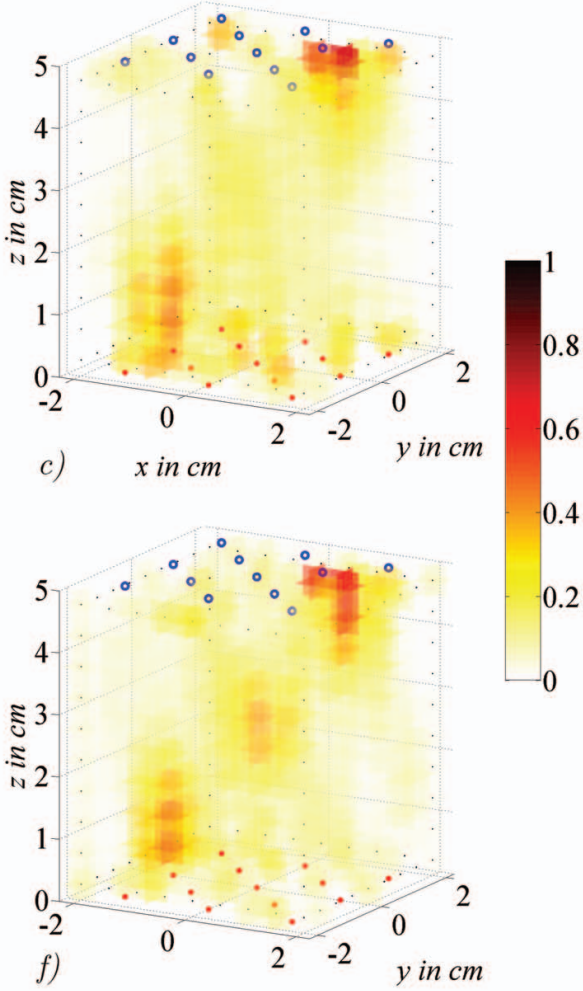

Fig. 1. Reconstructed concentration in the presence of $3 \%$ of gaussian noise. a) numerical phantom; b) reconstruction from CW signals; reconstruction from exponential B-splines MRA at: $c$ ) scale $3, d$ ) scale $2, e$ ) scale 1 , and $f$ ) scale 0.

\subsection{Reconstruction}

The inverse problem in FDOT is known to be ill-posed, meaning that a small perturbation in the data can lead to a large deviation in the reconstruction. To overcome this drawback, the inverse problem must be solved within the framework of regularization. Thus, the reconstructed vector $c^{*}$ is obtained by minimizing the Tikhonov cost function instead of the standard quadratic cost. Thus we have that:

$$
\boldsymbol{c}^{*}=\underset{\boldsymbol{c}}{\arg \min }\left\|\hat{\boldsymbol{m}}_{i}-\hat{\boldsymbol{W}}_{i} \boldsymbol{c}\right\|^{2}+\alpha\|\boldsymbol{c}\|^{2},
$$

where $\hat{\boldsymbol{m}}_{\boldsymbol{i}}$ and $\hat{\boldsymbol{W}}_{\boldsymbol{i}}$ are the MRA of $\boldsymbol{m}$ and $\boldsymbol{W}$ at scale $i$ as defined in (5) and (6); $\alpha$ is the regularization parameter. In practice, our implementation is based on the singular value decomposition of $\hat{\boldsymbol{W}}_{\boldsymbol{i}}$.

\section{NUMERICAL EVALUATION}

\subsection{Description of the synthetic phantom}

We consider the optically homogeneous infinite medium phantom shown in Fig. 1a). The absorption coefficient $\mu_{a}$ is set to $0.04 \mathrm{~cm}^{-1}$ and the reduced scattering coefficients $\mu_{s}^{\prime}$ to $9 \mathrm{~cm}^{-1}$. These values are in agreement with in vivo values of

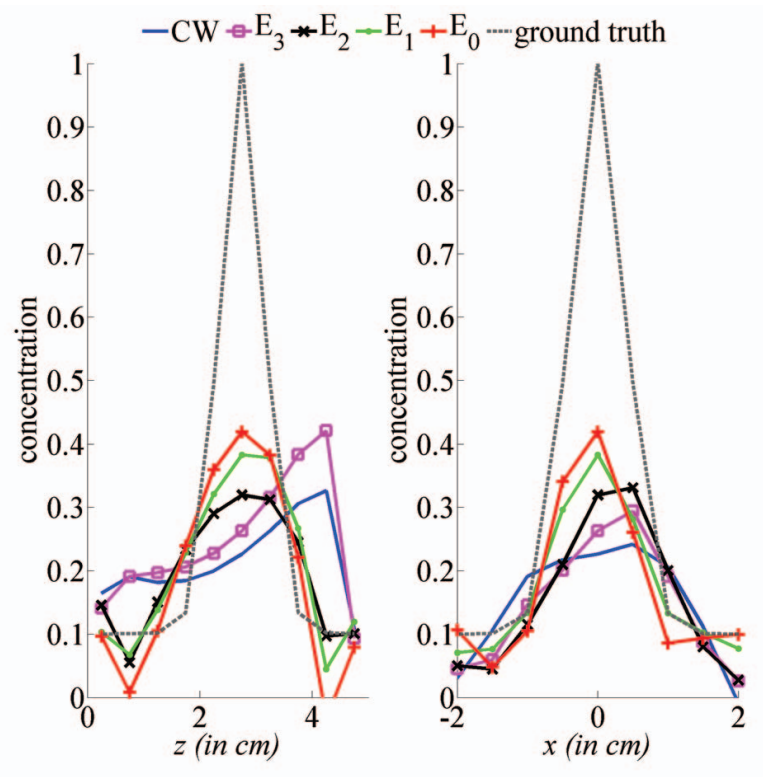

Fig. 2. Reconstructed concentrations around site number 2, along the $z$-axis (left) and the $x$-axis (right), for MRA scales $i=\{0,1,2,3\}$. 
breast tissue [12]. The homogeneity of the phantom allows to employ the analytical solutions of (1) [13]. The marker we use has a lifetime $\tau$ of $0.7 \mathrm{~ns}$ corresponding to the widely employed Alexa 750. The markers are concentrated preferentially around three sites at positions $\boldsymbol{r}_{\mathbf{1}}=[-1,-1,1.25]$, $\boldsymbol{r}_{\mathbf{2}}=[0,0,2.75]$, and $\boldsymbol{r}_{\mathbf{3}}=[1,1,4.25]$. To simulate the autofluorescence of the tissue as well as the nonspecificity of the markers, we also consider a fluorescence background with a background-to-site ratio of 1:10.

For the purpose of reconstruction, the medium is evenly discretized, resulting into 810 cubic voxels of size $0.5 \times 0.5 \times$ $0.5 \mathrm{~cm}^{3}$ covering a total volume of $4.5 \times 4.5 \times 5 \mathrm{~cm}^{3}$. The medium is excited by 13 point-sources and probed by 13 point-detectors. The sources and detectors are evenly arranged in two grids of side $3 \mathrm{~cm}$ placed $5 \mathrm{~cm}$ apart. Finally, the TR synthetic measurement vector $\boldsymbol{m}(t)$ is corrupted by $3 \%$ Gaussian noise.

\subsection{Results}

We present reconstructions of concentration vector in the geometry described in Section 4.1. In Fig. 1, we display the reconstructed concentration vectors $c^{*}$ for the MRA scales $i=\{0,1,2,3\}$. In Fig. 2, we show the reconstructed vectors along the $x$ and $z$-axis around the fluorescence site number 2 . It can be seen on both Fig. 1 and Fig. 2 that the poor quality of $\mathrm{CW}$ reconstruction can be enhanced by considering E-splines MRA reconstruction. Note that the reconstruction quality gets better when a finer MRA is considered.

\section{CONCLUSION}

We have proposed a new reconstruction scheme for TR FDOT that uses exponential spline basis functions. The proposed multiresolution E-spline framework is adapted to the representation of TR signals and provides a direct control of the resolution. The first results obtained on simulations show that this TR approach improves the reconstruction of deeply embedded markers compared with the $\mathrm{CW}$ approach. A detailed comparative study between the proposed TR approach and the gold-standard TR approach - based on the moments of the signals - is in progress.

\section{REFERENCES}

[1] E. Graves, J. Ripoll, R. Weissleder, and V. Ntziachristos, "A submillimeter resolution fluorescence molecular imaging system for small animal imaging," Med. Phys., vol. 30, no. 5, pp. 901-911, 2003.

[2] J. Huabei, "Frequency-domain fluorescent diffusion tomography: A finite-element-based algorithm and simulations," Appl. Opt., vol. 37, no. 22, pp. 5337-5343, 1998.
[3] J. Wu, L. Perelman, R. R. Dasari, and M. S. Feld, "Fluorescence tomographic imaging in turbid media using early-arriving photons and laplace transforms," Proc. Nat. Acad. Sci. U.S.A., vol. 94, no. 16, pp. 8783-8788, 1997.

[4] F. Gao, H. J. Zhao, Y. Tanikawa, and Y. Yamada, “A linear, featured-data scheme for image reconstruction in time-domain fluorescence molecular tomography," Opt. Express, vol. 14, no. 16, pp. 7109-7124, 2006.

[5] S. Lam, F. Lesage, and X. Intes, "Time domain fluorescent diffuse optical tomography: analytical expressions," Opt. Express, vol. 13, no. 7, pp. 2263-2275, 2005.

[6] N. Ducros, A. da Silva, J.-M. Dinten, and F. Peyrin, "Fluorescence diffuse optical tomography: A simulation-based study comparing time-resolved and continuous wave reconstructions performances," in IEEE ISBI, May 2008, pp. 388-391.

[7] J. Selb, A.M. Dale, and D.A. Boas, "Linear 3d reconstruction of time-domain diffuse optical imaging differential data: improved depth localization and lateral resolution," Opt. Express, vol. 15, no. 25, pp. 16400-16412, 2007.

[8] M. Unser and T. Blu, "Cardinal exponential splines: Part 1-theory and filtering algorithms," IEEE Transactions on Signal Processing, vol. 53, no. 4, pp. 1425$1438,2005$.

[9] A. Ishimaru, "Theory and application of wave propagation and scattering in random media," Proceedings of the IEEE, vol. 65, no. 7, pp. 1030-1061, July 1977.

[10] N. Ducros, A. da Silva, J.-M. Dinten, and F. Peyrin, "Approximations of the measurable quantity in diffuse optical problems: theoretical analysis of model deviations," J. Opt. Soc. Am. A, vol. 25, no. 5, pp. 1174-1180, 2008.

[11] M. S. Patterson and B. W. Pogue, "Mathematical-model for time-resolved and frequency-domain fluorescence spectroscopy in biological tissue," Appl. Opt., vol. 33, no. 10, pp. 1963-1974, 1994.

[12] T. Durduran, R. Choe, J. P. Culver, L. Zubkov, M. J. Holboke, J. Giammarco, B. Chance, and A. G. Yodh, "Bulk optical properties of healthy female breast tissue," Phys. Med. Biol., vol. 47, no. 16, pp. 2847-2861, 2002.

[13] S. R. Arridge, M. Cope, and D. T. Delpy, "The theoretical basis for the determination of optical pathlengths in tissue - temporal and frequency-analysis," Phys. Med. Biol., vol. 37, no. 7, pp. 1531-1560, 1992. 